FORMATION Formation emploi

Revue française de sciences sociales

101 | janvier-mars 2008

Numéro anniversaire : Regards croisés sur les

relations formation-emploi

\title{
De la flexicurité à la sécurité sociale professionnelle. L'emploi entre mobilité et stabilité
}

From flexicurity to occupational social security. Employment between mobility et stability

Flexicurity und "Sécurité sociale professionnelle » Beschäftigung zwischen Flexibilität und Sicherheit

De la flexiguridad a la seguridad social laboral. El empleo entre movilidad y estabilidad

\section{François Gaudu}

\section{OpenEdition}

Journals

Édition électronique

URL : https://journals.openedition.org/formationemploi/1052

DOI : 10.4000/formationemploi.1052

ISSN : 2107-0946

Éditeur

La Documentation française

Édition imprimée

Date de publication : 1 janvier 2008

Pagination : 71-88

ISSN : 0759-6340

Référence électronique

François Gaudu, « De la flexicurité à la sécurité sociale professionnelle. L'emploi entre mobilité et stabilité », Formation emploi [En ligne], 101 | janvier-mars 2008, mis en ligne le 31 mars 2010, consulté le 21 septembre 2021. URL : http://journals.openedition.org/formationemploi/1052 ; DOI : https:// doi.org/10.4000/formationemploi.1052 


\title{
Numéro anniversaire
}

\section{De la flexicuritél \\ à la sécurité sociale professionnelle. L'emploi entre mobilité et stabilité}

François Gaudu*

\begin{abstract}
"Sécurité sociale professionnelle » ou flexicurité ? Deux débats au contenu très proche se déroulent en France et en Europe. Dans ce contexte, l'article présente le débat français sur la réforme du droit du travail.
\end{abstract}

Lorsque j'ai écrit, dans Droit social, que la «Sécurité sociale professionnelle » constituait «un seul lit pour deux rêves» (Droit social, 2007), je voulais mettre en lumière le détournement de concept qu'a constitué le projet de «contrat de travail unique » (Cahuc et Kramarz, 2005), depuis semble-t-il devenu caduc (cf. infra "L'avatar $d u$ contrat de travail unique »). Fort habilement, ce projet de déréglementation du droit de la rupture du contrat de travail avait été présenté comme une forme de «Sécurité sociale professionnelle». Or la Sécurité sociale professionnelle, lancée dans le débat public par la CGT (Confédération générale du travail), ne visait certes pas à déréglementer...

Il est vrai que les deux notions de « flexibilité » et de " précarité » ne sont pas exactement des contraires. L'emploi peut être «flexible » sans être instable ou

${ }^{1}$ Cf. not. Liaisons Sociales Europe, ${ }^{\circ} 145$, « La magie s'opère autour de la flexisécurité »; ${ }^{\circ} 162$, «La flexicurité comme remède à tous les maux »; Jelle Visser (1997) ; LS Europe, n spécial, déc. 2005, «Le modèle social danois»; J.-C. Barbier (2005) ; J.-C. Barbier (2007). précaire - par exemple, dans le cas d'un salarié sous contrat à durée indéterminée, dont les horaires sont modulés et individualisés par un accord collectif ${ }^{2}$. Il n'empêche que le terme de flexicurité, contraction de flexibilité et de sécurité, est un oxymore ${ }^{3}$.

La contradiction est ainsi au cœur du sujet : contradiction dans les intentions; contradiction dans les effets supposés.

\footnotetext{
2 Art. L 212-8 C. trav.

${ }^{3}$ Oxymore : alliance de termes contradictoires (« Cette obscure clarté qui tombe des étoiles... »).
}

* François Gaudu est professeur à l'université Paris I Panthéon Sorbonne, Centre de recherches en droit social, Institut André Tunc. II a notamment publié : "La sécurité sociale professionnelle, un seul lit pour deux rêves », Droit Social, 2007.393 ; "Libéralisation des marchés et droit du travail », Droit Social, 2006.505. 


\section{- Contradiction dans les intentions}

L'un des premiers rapports qui ait proposé d'aller dans le sens de ce que l'on appelle maintenant « flexicurité » est le rapport Boissonnat (1995). Issu d'une commission composée majoritairement d'économistes, très divers par leurs orientations, ce rapport n'a pu voir le jour que parce qu'il parvenait à concilier des attentes contradictoires :

- Certains membres du groupe souhaitaient banalement un assouplissement du droit du travail, suivant la ligne d'argumentation qui avait servi, dans les années 80 , la revendication patronale d'une plus grande flexibilité de l'emploi ;

- D'autres se sont prononcés pour une évolution, non point parce qu'ils trouvaient le droit du travail trop contraignant, mais au contraire parce qu'ils regrettaient que la stabilité de l'emploi issue des années 70 ne constituât plus qu'un trompe-l'œil. En apparence, le droit n'a pas changé, mais en réalité, les carrières professionnelles sont devenues de plus en plus hachées, avec le développement des contrats précaires, et, pour ceux qui sont titulaires d'un contrat à durée indéterminée, la multiplication des licenciements pour motif économique. Il faut donc compenser une instabilité de facto excessive de l'emploi par les nouvelles sécurités d'un « droit des transitions ».

\section{- Contradiction dans les effets supposés}

Lorsque le salarié sait que l'assurance-chômage est généreuse et que les possibilités de formation et les moyens de reclassement sont consistants, il résiste d'autant moins au licenciement. C'est pourquoi le patronat trouve intérêt à l'existence de l'assurancechômage, que Gérard Lyon-Caen appelait la «législation auxiliaire du licenciement ».

Qu'on le veuille ou non, ce qui aide le chômeur, en rendant le licenciement plus supportable, aide à licencier. C'est pourquoi une critique « radicale» a souvent reproché au rapport Boissonnat, au rapport Supiot ou à la problématique des «marchés transitionnels » de s'accommoder de l'instabilité de l'emploi, voire de favoriser la remise en cause du droit du travail (cf. Coutrot, 1999; Lojkine, 1999). Cette critique était à la fois mal fondée et lucide :

- Mal fondée, car les projets en question ne comportaient pas de perspective de déréglementation. Au contraire, ils proposaient que des règles nouvelles soient introduites dans un domaine, celui des transitions professionnelles (de l'école au travail, d'une activité à une autre, du travail à la retraite...), qui échappait jusqu'alors largement au droit ;

- Lucide, car il est très clair, comme le montre l'exemple danois ${ }^{4}$, qu'une fois les transitions professionnelles organisées dans des conditions convenables, les attentes des salariés vis-à-vis du droit du licenciement se réduisent d'elles-mêmes. La simplification du régime de la rupture du contrat de travail devient ainsi plus facile.

Cela permet de comprendre pourquoi, sans duperie, des experts que l'on peut appeler respectivement « reréglementateurs » et "déréglementateurs» ont pu s'accorder, les uns cherchant à pallier l'inefficience de la réglementation, les autres escomptant les effets à long terme de l'innovation.

\section{- Un débat européen}

Ni la question, ni l'ambivalence, ne sont purement françaises. Le terme de flexicurité vient (si l'on peut dire...) de l'anglais flexicurity, utilisé successivement par les Hollandais et par les Danois pour rendre compte de leurs expériences. Le projet de contrat de travail unique trouve par ailleurs son pendant dans les propositions faites par la Commission européenne pour « moderniser le droit du travail» en novembre 2006 (Commission européenne, 2006). Quant au contenu réel des perspectives ouvertes, il y a tout lieu de croire que ce que les Anglais mettent sous le terme de flexicurity diffère fortement de ce qu'y placent des continentaux...

Les stratégies de flexicurité ne sont ainsi pas une, mais multiples. Aussi les voies d'évolution ouvertes par leurs mises en œuvre, si balbutiantes que soient celles-ci, sont-elles encore difficiles à discerner.

\footnotetext{
${ }_{4}^{4}$ S'il n'est pas vrai que le licenciement échappe au Danemark aux exigences de fond qui ont généralement cours en Europe continentale de l'Ouest ( $c f$. infra, "L'avatar du contrat de travail unique »), il semble en revanche exact que les Danois licenciés ont moins recours à la justice que d'autres.
} 


\section{LES STRATÉGIES DE FLEXICURITÉ}

La question a déjà une histoire. Les concepts aujourd'hui employés se sont constitués pendant la décennie qui va de 1993-1994 (lancement des travaux du groupe Boissonnat; prémices du document « Flexibility and security» diffusé en 1995 par le Gouvernement néerlandais) à 2002-2003 (la CGT adopte le mot d'ordre de "Sécurité sociale professionnelle »). Au moment des élections présidentielles françaises de 2007, la Sécurité sociale professionnelle figure dans le programme de la plupart des candidats - ce qui laisse à penser que la même expression recouvre des projets différents. Après la phase de genèse de la problématique s'ouvre ainsi celle des controverses.

\section{Genèse de la problématique}

Alors que les Français et les Allemands ont élaboré des concepts, les Hollandais et les Danois ont commencé à agir.

\section{L'innovation théorique : du contrat d'activité à la Sécurité sociale professionnelle}

Le thème de la Sécurité sociale professionnelle est passé par deux phases, que l'on pourrait appeler la phase des experts et la phase syndicale.

\section{- La phase des " experts»}

Pour que naissent des propositions nouvelles, il fallait d'abord abandonner l'illusion d'un possible retour en arrière, au temps heureux d'avant le premier choc pétrolier. Illusion perdue au début des années 90. Chacun peut alors constater que les propositions du rapport Auroux (1981)5 (« reconstituer la communauté de travail »...) et les lois qui lui ont fait suite n'ont ouvert aucune perspective stratégique nouvelle. Ébranlé par « la crise », le droit du travail ne sait plus où se trouve le progrès. Le statu quo n'a pas davantage de sens, puisque les dispositions en vigueur ne permettent pas d'empêcher le développement de la précarité, l'« éclatement de l'entreprise », etc.

\footnotetext{
${ }^{5}$ Le langage de la « restauration », à l'époque commun, est omniprésent.
}

L'ouverture de la discussion est facilitée par le fait que la Droite française, résultat de l'action de Monsieur Philippe Seguin au ministère du Travail, a provisoirement mis en sommeil, à partir de 1986, les thèses «déréglementatrices ». Le droit du travail connait ainsi une sorte de "répit dans la crise», qui conduit ceux qui l'osent à tenter d'imaginer des solutions pour rouvrir le jeu.

L'idée de Sécurité sociale professionnelle est inspirée par plusieurs rapports : rapport Boissonnat (1995), rapport Supiot (1999). Sans doute un économiste, plutôt que de se référer à ces rapports, évoquerait-il les «marchés transitionnels», en citant par exemple les travaux du sociologue allemand Günther Schmidt (2002) ou de Bernard Gazier (2003 ; 1998).

La proposition d'un renouveau du droit du travail part du constat précédemment évoqué que le contrat de travail classique ne suffit plus pour apporter aux salariés un degré suffisant - de sécurité matérielle, de maîtrise de leur avenir et d'identité professionnelle. Pour remédier à cette situation, il convient de raisonner dans un cadre plus vaste que celui de l'exécution d'un contrat isolé. Ce cadre plus vaste que le contrat de travail peut être :

- soit un contrat-cadre, susceptible d'englober plusieurs contrats de travail et, le cas échéant, plusieurs situations juridiques concomitantes ou successives. C'est le « contrat d'activité » du rapport Boissonnat, contrat d'au moins cinq ans passé avec un groupement (d'employeurs, d'organismes de formation, d'associations professionnelles...) qui garantit au travailleur la continuité de son activité par-delà des changements de position. La proposition trouve cependant sa limite dans le fait même qu'elle est fondée sur l'idée de contrat: tous les travailleurs, par conséquent, ne trouveront pas nécessairement de partenaire. Elle ne fournit ainsi qu'une solution partielle aux questions qu'elle veut résoudre ;

- soit une situation de type statutaire («statut de l'actif», "état professionnel des personnes»), englobant elle aussi, mais plus largement encore, un ensemble de situations juridiques par lesquelles un travailleur est susceptible de passer. Il s'agit alors de compléter le droit applicable au contrat de travail par un deuxième niveau de règles, non contractuelles (règles définissant un statut ou un «état»), qui 
expriment les droits et les devoirs du travailleur par rapport à la société, pour ainsi dire comme un effet de la citoyenneté.

\section{- La phase syndicale}

De 1996 à 1998, un séminaire dit « post-Boissonnat » s'est régulièrement réuni au Commissariat Général du Plan. Les partenaires sociaux, qui n'étaient pas représentés au sein du groupe Boissonnat, ont été invités à y participer.

Du côté patronal, le seul frémissement favorable est venu de la CGPME (Confédédation générale des petites et moyennes entreprises), qui a pris part à certaines réunions ${ }^{6}$. Le succès d'estime auprès des journalistes de la presse économique n'a donc pas eu de suite.

$\mathrm{Du}$ côté syndical, les réactions ont été très contrastées : réaction très hostile de Force ouvrière (FO). Cette opposition est notamment liée à la remise en question du rôle des «branches » qui résulterait d'une négociation sociale élargie, souvent «territorialisée ». Réaction froide de la CFDT (Confédération française démocratique du travail), mais pour une autre raison. La réticence porte moins sur le contenu des propositions que sur la méthode, qui fait jouer à l'État un rôle central. Aussi cette organisation se tient-elle à l'écart des travaux, en faisant savoir que les débats pertinents auront lieu, non au Commissariat du Plan, mais entre partenaires sociaux, dans le cadre de l'UNEDIC (Union nationale interprofessionnelle pour l'emploi dans l'industrie et le commerce). Réaction en revanche très favorable de la CGT. L'un de ses dirigeants, Jean-Christophe Le Duigou, prendra activement part à la plupart des réunions du séminaire « post-Boissonnat».

Il n'est donc pas surprenant que la CGT ait été le premier syndicat à reprendre à son compte les propositions du rapport Supiot. Après son congrès de 1999, elle se prononce en 2001 pour une « Sécurité sociale professionnelle » (voir Barbier et alii, 2002 ; Thibault,

\footnotetext{
${ }^{6}$ Cela s'explique peut-être par le fait que le rapport Boissonnat portait attention au travail non salarié, alors que la tradition des analyses « sociales », jusqu'à ce rapport et à l'ouvrage de Gérard Lyon-Caen (Le droit du travail non salarié, Dalloz, 1997), était de ne traiter plus que des salariés.
}

2002). Quoique le contenu de celle-ci ne soit pas très précisément défini ( $c f$. toutefois Le Duigou, 2005), on peut dire que l'orientation comporte deux faces :

- Sous un angle, il s'agit d'une réaffirmation de la stabilité de l'emploi. Pas de licenciement sans reclassement préalable : l'objectif est une extension de ce que l'on pourrait appeler le modèle de la fonction publique, perspective assez traditionnelle ;

- D'un autre côté, on ne présente plus les passages d'un emploi à un autre comme des événements nécessairement subis par les salariés. Le droit doit aider le salarié à aller d'un moins bon emploi vers un meilleur. Ainsi, la préservation de l'emploi tel qu'il est cesse d'être l'alpha et l'oméga de la politique syndicale - ce qui conduira notamment la CGT à revendiquer avec force la transférabilité des droits d'une entreprise à une autre.

L'appellation de «Sécurité sociale» présente un avantage sur le plan de la «communication», puisqu'elle évoque un système déjà existant auquel le monde salarial est attaché. Cet avantage est payé d'un inconvénient conceptuel: la Sécurité sociale assure une couverture contre des risques, suivant un mécanisme qui demeure marqué par l'idée d'assurance. Or, l'un des aspects les plus modernes des positions de la CGT est de vouloir fournir aux salariés des outils qui leur permettent d'organiser leur carrière, indépendamment de la réalisation d'un «risque de l'emploi». Dans cette perspective «proactive», l'idée de Sécurité sociale n'a plus vraiment sa place.

Preuve qu'il a été bien choisi, c'est néanmoins le vocable de Sécurité sociale professionnelle qui va s'imposer lorsque la discussion entrera dans sa phase politique.

\section{Les innovations pratiques du Nord de l'Europe}

Les Danois ayant été de meilleurs communicateurs que les Néerlandais, c'est du «modèle danois» qu'il est le plus souvent question. Mais on peut compter sur les Pays-Bas pour faire valoir leur droit d'antériorité.

\section{- La Hollande}

L'une des différences essentielles entre la Hollande et la France tient au fait que les réformes du droit du 
travail y ont, à plusieurs reprises, fait l'objet d'accords de principe entre le patronat et les syndicats. C'est ainsi que l'accord de Wassenaar a mis en place, dès 1982, un cadre négocié organisant de pair modération salariale et réduction du temps de travail (cf. Institut de l'entreprise, 1998).

Confrontés à la récession du début des années 90 , les Pays-Bas ont, au terme d'âpres débats ${ }^{7}$, pris le parti de favoriser le développement du travail à temps partiel et de diverses formes d'emploi atypiques (Jelle Visser, 1999), tout en assurant une meilleure protection aux salariés titulaires de ces emplois. C'est ainsi, par exemple, que l'équivalent des heures complémentaires ${ }^{8}$ peut faire l'objet de majorations de salaires dans les mêmes conditions que les heures supplémentaires, ou encore que les contrats précaires sont limités dans le temps ${ }^{9}$. Le travailleur temporaire est lié à l'entreprise de travail temporaire par un contrat de travail ${ }^{10}$, qui peut être à durée indéterminée. Avec l'accord de représentants des salariés, les contraintes légales en matière de temps de travail peuvent être aménagées - mais plus de la moitié des salariés à plein temps, en 1999 , sont à 36 heures par semaine ; l'individualisation des horaires s'est développée - mais une loi du 8 février 2000 permet au salarié de demander l'augmentation ou la diminution de la durée du travail, l'employeur ne pouvant refuser qu'en justifiant d'un intérêt sérieux ${ }^{11}$. C'est cet équilibre entre flexibilité de l'emploi et droits des salariés audacieusement améliorés qui donne naissance au terme de « flexicurité »

Quant au licenciement, encore qu'il ait fait l'objet de quelques mesures d'assouplissement en 1998, il reste très réglementé : il requiert en principe une autorisation administrative, à moins que - pour gagner du

\footnotetext{
${ }^{7}$ Document gouvernemental sur « la flexibilité et la sécurité » de 1995, conduisant, avec l'accord des partenaires sociaux, à une loi adoptée en 1998.

${ }^{8}$ Heures accomplies par les salariés à temps partiel en sus de celles normalement prévues.

${ }^{9}$ Comme en France, leur renouvellement excessif les transforme en contrat à durée indéterminée.

${ }^{10} \mathrm{La}$ solution est la même en France, mais elle est discutable, puisque le travailleur temporaire œuvre en réalité pour le compte de l'entreprise utilisatrice.

${ }^{11}$ V. Susanne Burri, «Temps de travail à la carte», Liaisons Sociales Europe, n ${ }^{\circ}$, p. 5, fév. 2000. Un bilan ayant été dressé, la loi a été maintenue (v. Liais. Soc. Europe, n ${ }^{\circ}$ 122, 17 fév. 2005, p. 6).
}

temps - l'employeur ne sollicite la résolution judiciaire pour une cause sérieuse (serious cause).

Les Hollandais ont réduit leur taux de chômage, très largement, en faisant sur le marché du travail place aux femmes, auparavant très sous-employées, grâce au temps partiel.

\section{- Le Danemark}

La répercussion de l'expérience danoise tient en partie à d'excellents résultats sur le terrain du taux d'activité. La société danoise présente certes des points communs avec la société hollandaise: les réformes se négocient entre patronat et syndicats. Mais elle présente aussi de fortes différences culturelles : comme la Suède, le Danemark exerce une forte pression sociale sur ses citoyens pour qu'ils adoptent un comportement considéré par le groupe comme acceptable. C'est pourquoi, au lieu de fonder comme la Hollande sa stratégie sur l'essor de contrats atypiques réglementés - solution individualiste -, il a privilégié la mise en place de politiques actives de l'emploi très redistributives : très bonne couverture par l'assurance-chômage - jusqu'à quatre ans à un niveau élevé d'indemnisation; service de l'emploi, notamment de placement, très efficace, récemment réformé dans le cadre d'une décentralisation accompagnée de responsabilités financières accrues pour l'échelon municipal (le nombre de municipalités devait passer de 250 à 100, et l'échelon des comtés être supprimé, pour permettre, entre autres, cette réforme); formation ( « rééducation pour l'emploi ») très ambitieuse.

Les croyances collectives, ici, ont presque plus d'importance que les contraintes juridiques: une pression sociale s'exerce sur les individus pour qu'ils exercent une activité utile; elle engendre une pression sur les entreprises pour que celles-ci fassent place à tous. Chacun, sachant ce qu'est la pression sociale, accepte de payer pour financer le système : c'est, je crois, le cercle vertueux de la confiance. De ce point de vue, les commentaires « déçus » sur les spécificités des politiques d'immigration scandinaves, et spécialement danoise ${ }^{12}$, sont eux-mêmes

${ }^{12} C f$. Barbier, op. cit., dir. Paugam, p. 11, qui parle de «face d'ombre »; R. Boyer, op. cit., range parmi les «faiblesses» danoises le bas taux d'activité des étrangers (p. $42 \mathrm{~s}$.) 
surprenants: les politiques scandinaves reposent dans une très large mesure sur la cohésion d'un groupe très homogène $\mathrm{e}^{13}$. Il est tout à fait logique que ces pays réagissent de façon beaucoup plus rapide et énergique que la France lorsqu'ils constatent ou croient constater qu'un sous-groupe adopte un comportement de «passager clandestin $»^{14}$.

\section{Les controverses : les deux flexicurités}

Aux élections présidentielles de 2002, le thème de la Sécurité sociale professionnelle n'apparaît que dans les programmes de «petits candidats ». Alors que les élections de 2007 s'annoncent, celle-ci figure au programme officiel du Parti socialiste; elle constitue aussi un «élément de langage» du candidat de I'UMP (Union pour un mouvement populaire) ${ }^{15}$.

Que s'est-il passé, entre 2002 et 2007, qui explique cette confondante convergence, au moins verbale ?

Le Parti socialiste n'avait plus, dans son fonds de doctrine, d'équivalent de ce qu'avait représenté le « partage du travail ». Il est donc logique qu'il se soit saisi de la perspective offerte. Encore la traduction qui en est faite est-elle assez banale: il s'agit de « relooker» des formules, depuis longtemps communes aux hauts fonctionnaires chargés de la politique de l'emploi ${ }^{16}$ - formation tout au long de la vie, guichet unique pour les chômeurs ${ }^{17} \ldots$ Lorsque François Fillon était ministre du Travail, conseillé par les mêmes hauts fonctionnaires, il avait lui-même

\footnotetext{
${ }^{13}$ Ce qui explique les «civic attitudes » (attitudes citoyennes) relevées par Yann Algan et Pierre Cahuc (2005).

${ }^{14}$ Cf. l'Aliens act danois de 2002 (texte en anglais sur http :// www.inm.dk et aussi Centre d'Analyse Stratégique (2006).

${ }^{15}$ V. N. Sarkozy, « Retrouvons le plein emploi grâce à la Sécurité sociale professionnelle », La Tribune, 12 décembre 2006.

${ }^{16}$ La notion de «parcours" - parcours de formation, parcours d'insertion, parcours professionnel... - est apparue dans les années 80, en premier lieu dans l'ordonnance Schwartz du 26 mars 1982 (cf. Gaudu, « Du statut de l'emploi au statut de l'actif», Droit Social $\left.95.538, \mathrm{n}^{\circ} 16\right)$. On passe, si l'on ose dire, par glissements, du parcours aux «trajectoires» au début des années 90. V. not. «Transitions et trajectoires », Observatoire de l'ANPE, Troisièmes entretiens de l'emploi, 19-20 mars 2003; D. Méda, B. Midault, «La sécurisation des trajectoires professionnelles», Document d'études DARES, $\mathrm{n}^{\circ} 107$, oct. 2005. La loi sur la participation du 30 déc. 2006 comporte une partie consacrée à la « sécurisation des parcours professionnels ».

${ }^{17}$ Projet socialiste pour la France, partie I, « Construire avec les partenaires sociaux une sécurité professionnelle ».
}

fait un pas dans le même sens en utilisant le vocable $\mathrm{d}^{\prime}$ «assurance-emploi $»^{18}$.

Le rattachement de la Sécurité sociale professionnelle à des problématiques déjà classiques est abandonné dans les propositions que fait Monsieur Nicolas Sarkozy, alors candidat de l'UMP. Celui-ci reprend en effet à son compte la proposition de «contrat de travail unique » issue du rapport Cahuc-Kramarz, et avec celle-ci, il adopte l'emprunt rhétorique qu'a fait ce rapport de l'expression de Sécurité sociale professionnelle. Dans une orientation très différente de celles qui ont été jusqu'à présent évoquées, il s'agit de déréglementer, tout en le taxant, le licenciement pour motif économique et de réformer le service public de l'emploi (Cahuc, Kramarz, 2005 ; Gaudu, 2005).

Il est assez généralement admis que le degré de protection de l'emploi est sans incidence prouvée sur le taux de chômage (voir notamment Gautié, 2005). C'est un autre argument, l'effet discriminatoire que celle-ci comporterait, qui fonde la proposition de contrat de travail unique. La stabilité de l'emploi ferait naître des «inégalités de traitements » qui défavorisent les moins bien lotis (jeunes, seniors) (Cahuc, Kramarz, p. 14, 2005) : "plusieurs dizaines d'études empiriques menées dans divers pays, avec des données mobilisant des milliers d'observations indiquent que la protection de l'emploi est plutôt défavorable à l'emploi, en particulier pour les groupes démographiques, tels que les jeunes, les femmes et les travailleurs agés, dont l'insertion sur le marché du travail est la plus difficile. » (Cahuc, Kramarz, 2005, p. 158)

Les articulations du rapport Cahuc-Kramarz se retrouvent toutes dans le Livre vert pour la modernisation du droit du travail proposé par la Commission européenne en $2006^{19}$. L'idée de l'« exception française», fondement du rapport, ne résiste ainsi pas à l'examen. En réalité, il y a deux débats parallèles - un débat

\footnotetext{
${ }^{18}$ Intervention devant l'Assemblée nationale, 4 février 2003. V. aussi « Les nouvelles orientations des politiques de l'emploi à la mi-2002 », ministère du Travail, Infos Europe, http://www.travail.gouv.fr/liens_euro.html.

19 (1) Il y a des insiders et des outsiders sur le marché du travail ; (2) Cette segmentation est liée au développement d'une « flexibilité marginale », autrement dit des contrats précaires; (3) Cette segmentation produit des effets discriminatoires ("Le risque d'être en position de faiblesse sur le marché du travail comporte aussi une forte dimension de genre et de génération " : soit de sexe et d'âge...).
} 
français et un débat européen -, qui mettent aux prises des positions sensiblement identiques, et il y a deux conceptions de la flexicurity en Europe.

\section{L'avatar du contrat de travail unique}

\section{- Le contenu du « contrat de travail unique»}

La réforme que propose le rapport Cahuc-Kramarz peut être présentée ainsi :

- I. Le contrat de travail à durée déterminée et le contrat de travail à durée indéterminée fusionnent dans un « contrat unique », à durée indéterminée.

- II. Il n'y a donc plus de contrat à terme (et la stabilité, toute limitée qu'elle soit, du contrat à durée déterminée ${ }^{20}$, disparaît par conséquent).

Pour autant, le nouveau contrat à durée indéterminée n'est pas un contrat stable. En effet, il y a :

- III. Supression du contrôle du motif économique de licenciement. Le droit distingue le licenciement pour motif personnel du licenciement pour motif économique. En cas de licenciement pour motif personnel, le contrôle du juge sur le caractère réel et sérieux du motif actuellement prévu par la loi serait maintenu. En revanche, le contrôle du juge sur le motif économique doit disparaître. En effet, « les pouvoirs publics ne disposent pas de l'information nécessaire pour évaluer l'efficacité de chaque licenciement sur la base de critères économiques. [...] le contrôle des motifs économiques [...] est aléatoire du fait de l'hétérogénéité des critères des juges et du talent des avocats. Il repose sur une information insuffisante et demande des délais très importants en cas de litige $[. ..] »^{21}$.

\footnotetext{
${ }^{20}$ L'employeur ne peut en effet rompre le contrat à durée déterminée qu'en cas de faute grave ou de force majeure (art. L 122-3-8 C. trav.).

${ }^{21}$ Rapport Cahuc 2003, pp. 38-39. Rappr. Blanchard et Tirole, Protection de l'emploi et procédures de licenciement, rapport pour le CAE, 2003, p. 47 : «Le licenciement pour cause économique doit [...] donner droit aux allocations chômage pour le ou les salariés licenciés, et au paiement de taxes et indemnités de licenciement par l'entreprise. Ces paiements responsabilisent l'entreprise face aux licenciements. Si, dans ces conditions, l'entreprise est prête à effectuer ces paiements, nous ne saisissons pas la logique sous-jacente à l'examen et à l'invalidation de cette décision par l'appareil judiciaire. On peut penser que le système en place à l'heure actuelle, qui permet aux juges de contester la décision de licenciement de l'entreprise, trouve sa justification dans l'absence de responsabilisation financière des entreprises. La solution nous paraît être de responsabiliser les entreprises, non de demander aux juges de contester la décision de l'entreprise. »
}

- IV. Suppression des obligations de reclassement prévu en cas de licenciement économique (obligation de reclassement interne dans l'entreprise et le groupe, plan de sauvegarde de l'emploi - ex «plan social »pour les « grands licenciements »).

- V. Remplacement de l'un et de l'autre par une incitation financière (indemnité de licenciement majorée et/ou taxation du licenciement). On parle de contrat « à consolidation progressive» parce que le coût de la rupture - l'indemnité de licenciement croît avec l'ancienneté.

\section{- Abandon du projet de contrat de travail unique}

Le projet de contrat de travail unique a été très présent dans la communication gouvernementale immédiatement après l'élection présidentielle. Il figure dans la déclaration de politique générale présentée par le Premier ministre à l'Assemblée nationale le 3 juillet 2007 .

Force est cependant de constater que, depuis lors, le contrat de travail unique a disparu de l'agenda gouvernemental. Il n'en est ainsi question, ni dans la Lettre de mission de Madame Christine Lagarde, ministre chargée de 1'Emploi (11 juillet 2007), ni dans la Lettre de mission de Monsieur Xavier Bertrand, ministre du Travail (1 ${ }^{\text {er }}$ août 2008), ni dans le communiqué du ministère de l'Économie, des Finances et de 1'Emploi présentant la Commission pour la libération de la croissance française («Commission Attali », août 2007).

À l'issue de la négociation de l'accord national interprofessionnel (ANI) sur la «modernisation du marché du travail » du 11 janvier $2008^{22}$, il est clair que le projet de contrat de travail unique s'est brisé sur l'opposition des partenaires sociaux.

\section{- Pourquoi le contrat de travail unique a-t-il été rejeté?}

Pourquoi les partenaires sociaux se sont-ils opposés au contrat de travail unique? L'opposition des syndicats de salariés se passe presque d'explication, puisqu'il s'agit d'une remise en cause de la stabilité de l'emploi. Leur position rejoint en cela les

${ }^{22}$ Liaisons Sociales, $\mathrm{n}^{\circ}$ 22/2008. 
réactions de la Confédération européenne des Syndicats face au Livre Vert proposé par la Commission européenne ${ }^{23}$. L'opposition du patronat obéit sans doute à deux raisons :

- La préoccupation de ne pas lâcher la proie pour l'ombre est sans doute l'une d'entre elles. Le contrat à durée déterminée présente pour l'employeur un avantage juridique, avec la rupture automatique du contrat par la survenance du terme. Peut-être la possibilité de licencier pour motif économique, sans indemnité de précarité ni possibilité de contrôle du motif par le juge, est-elle sur le papier encore plus avantageuse. Mais il faudra néanmoins licencier... Or les obstacles concrets au licenciement et le contenu de la législation sont jusqu'à un certain point distincts.

Ainsi, la loi japonaise est, en matière de licenciement, plutôt moins contraignante que les lois européennes; il est cependant généralement admis que le licenciement des salariés «typiques $»^{24}$ y est plus difficile qu'en Europe, parce que la société japonaise n'admet pas ces licenciements ${ }^{25}$. En d'autres termes, le licenciement rencontre des obstacles non juridiques. Et il en va de même en France : l'annonce de licenciements peut être un facteur d'inquiétude dans l'entreprise, alors que la survenance du terme d'un contrat à durée déterminée et le départ du salarié intéressé seront ressentis comme des événements normaux. Le contrat de travail unique, même très peu réglementé, aurait privé le patronat de l'avantage « sociologique » que procure le contrat à durée déterminée.

- "Chat échaudé craint l'eau froide » est sans doute la seconde explication. Le patronat vient de faire l'expérience juridique du «contrat nouvelle embauche » (CNE) créé par l'ordonnance du 2 août 200526. Ce contrat permettait le licenciement sans

\footnotetext{
${ }^{23}$ V. la position adoptée par le Comité exécutif de la CES à Rome les 20 et 21 mars 2007 (http ://www.etuc.org/a/3556), ou encore la réaction de John Monks à la Communication sur la flexicurité de la Commission européenne du 27 juin 2007, qui regrette que l'objectif de "donner aux entreprises davantage de liberté de licencier [...] semble être l'idée maîtresse de la communication » (http://www.etuc.org/a/3768).

${ }^{24}$ De sexe masculin, Japonais et qualifiés...

${ }^{25}$ En 2005, l'ancienneté moyenne dans l'emploi est ainsi de 12,2 années au Japon, contre 10,7 dans l'Union européenne (et 11,7 en France) (P. Auer, B. Gazier, L'introuvable sécurité de l'emploi, Flammarion, 2006).

${ }^{26}$ Ordonnance $\mathrm{n}^{\circ} 2005-893$.
}

contrôle judiciaire du motif pendant les deux premières années. Qu'a-t-on vu à cette occasion?

La France a ratifié la convention $n^{\circ} 158$ de l'Organisation internationale $\mathrm{du}$ travail (OIT) sur le licenciement, et les conventions internationales régulièrement ratifiées ont, d'après l'article 55 de la Constitution, une autorité supérieure à la loi. Or, d'après la convention 158 de l'OIT, le licenciement n'est possible que s'il existe un «motif valable $»^{27}$. Cette exigence peut être écartée pendant la période d'essai ou pendant une période d'une durée «raisonnable ${ }^{28}$. Mais la Cour d'appel de Paris a jugé, dans un arrêt du 6 juillet 2007, que la période de deux ans pendant laquelle le droit du licenciement se trouvait mis à l'écart était à cet égard excessive. En outre, le Conseil d'administration du Bureau international du travail (BIT) a estimé, dans un rapport du 14 novembre 200729 , que la période de consolidation du CNE - deux ans - n'était pas d'une « durée raisonnable».

L'échec juridique du CNE a conduit le patronat à y renoncer dans l'accord du 11 janvier 2008, et cet échec, bien évidemment, a pesé sur le projet de contrat de travail unique. Toutefois, si la convention $\mathrm{n}^{\circ} 158$ de l'OIT n'existait pas, les juristes se seraient rabattus sur l'article 1780 du Code civil, qui prévoit que le juge peut octroyer des dommages-intérêts au salarié licencié suivant les circonstances, c'est-à-dire en cas de licenciement abusif. Et même sans ce texte, les juges pourraient condamner l'employeur en se fondant sur la théorie générale de l'abus de droit.

- Raisons théoriques de l'échec : c'est une vue de l'esprit de vouloir chasser le juge du droit du licenciement

L'intervention du juge, suivant les économistes néoclassiques, ne se justifie pas lorsqu'elle porte sur le motif économique - réserve faite de la qualification de celui-ci.

Le juge, disent Cahuc et Kramarz, n'a pas l'information nécessaire pour apprécier le motif d'un licenciement économique. Mais, objectai-je dans une

\footnotetext{
${ }^{27}$ Art. 4.

${ }^{28}$ Art. 2.

${ }^{29}$ Liaisons Sociales, Bref social, 19 nov. 2007.
} 
discussion, en droit de la concurrence, dans tous les pays, comme en droit communautaire de la concurrence, les concentrations d'entreprises sont interdites ou autorisées au terme d'évaluations tout aussi « imprécises » que les jugements portés sur le motif économique d'un licenciement. Ces décisions se font, dans une certaine mesure, lorsque la solution n'est pas flagrante, «au doigt mouillé ». Elles intègrent bien évidemment des données politiques. Elles comportent fréquemment des aspects de mise en balance des intérêts, notamment lorsqu'une concentration assez forte peut être justifiée par les avantages économiques ou sociaux qu'elle comporte. Ainsi, l'on n'hésite pas à faire dépendre le sort d'entreprises ou de groupes (avec à la clé des risques économiques pour les acteurs, en cas de refus, sans doute supérieurs à ceux qui naissent d'une condamnation pour licenciements irréguliers) de décisions « approximatives » d'un juge ou d'une autorité administrative.

Je pensais l'argument propre à convaincre des économistes : si le droit du travail «fait comme» le droit de la concurrence, on n'est donc pas en présence d'une « fantaisie » sociale qui traduirait les préoccupations d'une époque révolue. Cet argument n'a apparemment eu aucun effet. On peut en conclure que, pour les économistes néo-classiques, les décisions des autorités de concurrence ne seraient légitimes que si elles se bornaient à reprendre les « acquis de la théorie économique ». Dans la mesure où elles font bien plus, elles sont tout aussi illégitimes que le contrôle du juge français sur le motif du licenciement économique.

C'est donc moins le droit français du licenciement économique que le principe de la mise en balance des intérêts qui se trouve sur la sellette. Or, s'il y a un point sur lequel des juristes de différents pays et de toutes spécialités s'entendraient volontiers, c'est certainement la force d'expansion considérable du principe de proportionnalité.

La mise en balance des intérêts, dans les licenciements économiques, prend deux formes, le contrôle du caractère réel et sérieux du motif d'une part, le contrôle des efforts de reclassement accomplis par l'employeur, d'autre part. Sur ces deux terrains, le juge, loin de vérifier, comme on le dit parfois, l'opportunité des décisions prises, applique le principe de proportionnalité (le licenciement d'un salarié qui coûte trop cher dans une entreprise où les profits sont considérables n'a pas de motif économique $^{30}$, énoncé surprenant de prime abord, mais qui exprime clairement le contrôle de proportionnalité qu'a exercé le juge : il ne faut pas que le dommage subi par le salarié, par hypothèse substantiel, soit sans commune mesure avec l'avantage économique minime que l'entreprise retire du licenciement). Plus généralement, dans les sociétés européennes, lorsque la situation professionnelle d'une personne dépend de la décision unilatérale de son cocontractant, le droit tend assez souvent à ce que cette décision ne puisse pas être prise de façon arbitraire. D'où la mise en balance des intérêts: le motif qui conduit à prendre une décision de rupture ne doit pas être dérisoire au regard des inconvénients subis par le partenaire. Celui qui rompt le contrat - de mandat, de bail commercial, de travail... - doit disposer d'un motif, et il doit parfois énoncer ce motif, indépendamment de tout contentieux, comme c'est le cas en matière de licenciement ${ }^{31}$.

L'affirmation du principe de proportionnalité, c'està-dire du pouvoir de contrôle du juge, n'est pas une tendance seulement française, mais européenne. Par exemple, contrairement à ce que l'on croit pouvoir écrire un peu partout en France, en droit du travail danois, les conventions collectives prévoient en général ${ }^{32}$ que le licenciement doit être justifié par un «motif raisonnable». À défaut, la loi prend le relais $^{33}$. Le Danemark ne soumet pas la rupture du contrat de travail à une théorie de l'employment at

\footnotetext{
${ }^{30}$ Soc. 24 avr. 90, B. $\mathrm{n}^{\circ} 183$.

${ }^{31}$ Le non-renouvellement du bail commercial doit être motivé (art. L 145-9 s. C. Com.). Sur la motivation en droit privé, v. Revue des contrats, $\mathrm{n}^{\circ} 2,2004$.

32 Selon la Fondation de Dublin (http://eurofound.europa.eu/emire/ DENMARK/UNFAIRDISMISSAL-DN.html), " in Denmark, it is stipulated by the relevant collective agreements (for example, $\$ 4$ (3) of the DA/LO Basic Agreement...)... that dismissal must be justified by a reasonable cause (saglig grund) relating to the employee or to factors concerning the enterprise ». V. aussi A. Lefebvre, «Le modèle social danois", Liaisons Sociales Europe, déc. 2005, p. 35 s.

${ }^{33}$ Le licenciement doit être «raisonnablement justifié par le comportement $d u$ salarié ou la situation de l'entreprise» (the Employers' and Salaried Employees '(Legal Relationship) (Consolidation) Act, 1996, Act n ${ }^{\circ}$ 642, af. 28 June 1996, trad. FG, d'après la version anglaise de la loi accessible sur le site natlex de l'OIT).
} 
will $^{34}$. La voie danoise est ainsi très différente de celle d'une déréglementation.

Malgré tous les efforts (rhétoriques) faits pour rattacher ses propositions au contexte du droit du travail français, il me semble que le rapport Cahuc-Kramarz présente une pensée atemporelle. En réalité, l'état du droit français importe peu à sa démonstration ${ }^{35}$. La doctrine économique néo-classique, dans l'état qui est le sien actuellement - c'est peut-être une ombre portée de la théorie des asymétries d'information croit qu'il est irrationnel que des jugements de valeur soient portés de l'extérieur sur les choix économiques individuels. Soit les décisions du juge sont assujetties au consensus de la théorie économique ; soit elles doivent être remplacées par des incitations, elles-mêmes instituées suivant ledit consensus.

De façon cohérente avec ce qui précède, après avoir montré que la France et l'Allemagne n'exercent qu'un contrôle très faible sur le comportement des demandeurs d'emploi ${ }^{36}$, ils n'adoptent cependant pas non plus l'idée que ce comportement devrait être examiné de plus près. La dégressivité des allocations leur paraît une solution préférable. Le chômeur et le chef d'entreprise sont ainsi traités de la même façon : on n'est donc pas en présence d'une doctrine « patronale», mais plutôt d'une idéologie disciplinaire. Remplacer les notions floues, qui réclament interprétation, donc jugement, par des incitations, c'est en somme mettre la technique à la place du droit. Précisément, cela n'est pas possible et une théorie qui prétend agir ainsi, méconnaissant «l'épaisseur du droit», donc la réalité, n'est donc pas « scientifique ».

\footnotetext{
${ }^{34}$ L'arbitrage entre durée du préavis et montant des dommagesintérêts en cas de rupture irrégulière étant cependant inverse qu'en France : plus long préavis, réparation à un niveau plus faible.

${ }^{35}$ Lorsque Daniel Cohen est interviewé par «Le Monde » sur le droit du licenciement (10 janv. 2008), il commet aussitôt une bourde («On ne peut licencier que pour deux raisons : pour motif économique et pour faute »), puisque l'on peut aussi entre autres licencier pour inaptitude physique ou pour insuffisance de résultats. De même, la partie juridique du rapport BlanchardTirole (préc.) est remplie d'erreurs. Le plus amusant est que cela n'a sans doute aucune importance: ils arrivent avec cela aux mêmes conclusions que Pierre Cahuc (qui, lui, fait attention à ce qu'il dit du droit), puisque c'est la théorie économique qui dicte les conclusions, et non la considération du monde réel. 36 P. 64.
}

\section{La voie anglaise et la voie continentale}

Une façon de désigner l'«au-delà de l'emploi » consiste à dire qu'il faut protéger la personne, et pas l'emploi. La formule, que Pierre Cahuc et Francis Kramarz ont pour ainsi dire empruntée à la CGT $^{37}$, possède une force incontestable.

Mais que veut-on dire par «protéger la personne»? Il semble qu'il y ait, au moins, deux sens concevables :

- Il est possible de protéger la personne dans son rapport au marché. Il s'agit alors d'« armer » le travailleur pour la grande lutte sur le marché du travail ${ }^{38}$. On tombe alors logiquement sur les quatre ingrédients de la politique de l'emploi "whig» anglaise: formation professionnelle + service de l'emploi, l'un et l'autre, idéalement, rendus efficaces + politique volontariste de lutte contre les discriminations + assurance-chômage minimaliste ${ }^{39}$. C'est, à peu de choses près (sauf le dernier point ?), ce que propose le rapport Cahuc-Kramarz ;

- Mais il est possible également de protéger la personne, en considération de son appartenance à un groupe. Alors, la sécurisation des trajectoires professionnelles ne se résume pas à armer l'individu pour le (fair) struggle for life (lutte pour la vie). « Socialisation plus large des droits et des responsabilités » (Le Duigou, op. cit., p. 48), elle devient un effet de la citoyenneté ou, en tout cas, elle traduit un rapport d'appartenance - statut de l'actif, état professionnel... La société doit à l'individu plus que la formation et le placement sur un marché non déloyal. Elle lui doit un « état » et, lorsque celui-ci ne peut plus se confondre avec un « emploi à vie », l'état professionnel résulte

\footnotetext{
${ }^{37}$ Comp. J.-C. Le Duigou, op. cit., p. 48, et Cahuc, Kramarz, préc. Dans sa présentation de la résolution générale sur l'orientation au 46 ${ }^{\mathrm{e}}$ Congrès de la CFDT (Liaisons Sociales, Syndicats 01/07, p. 7 s), Marcel Grignard reprend une formulation assez proche. Si bien que, malgré l'affichage de différences de sensibilités entre la CGT et la CFDT, je ne suis à ce stade de la discussion pas certain qu'une divergence soit vraiment constituée. Comp. rapport d'orientation du $48^{\mathrm{e}}$ Congrès de la CGT, Liaisons Sociales W 421, p. 12. En réalité, les positions de chacune des deux confédérations comportent des ambiguités, et ce ne sont pas les mêmes...

${ }^{38} C f$., à propos de la flexicurité, Gazier et Auer, op. cit., p. 128.

${ }^{39}$ Dans les faits, en Grande-Bretagne, à un niveau plus proche du RMI (revenu minimum d'insertion) que des allocations versées par les ASSEDIC (contribution based income à 57,45 livres, soit 85,81 euros par semaine pour une personne seule âgée de plus de 25 ans ; le RMI français est à 389,10 euros par mois...).
} 
du fait que l'individu dispose de marges de manœuvre consistantes pour planifier sa carrière et de points de repère institutionnels. C'est le système danois : outre les quatre ingrédients anglais, (1) le groupe finance quatre ans d'indemnisation du chômage, ce qui est plus que de rendre la force de travail vendable de façon expéditive ; (2) le marché du travail est encadré par une négociation collective équilibrée, et les entreprises sont soumises à un système de cogestion (Jackson, 2005). De même, aux Pays-Bas, une réglementation étatique protectrice complétée par une négociation collective équilibrée vient encadrer les choix qui peuvent être faits sur le marché.

La France est un pays plus grand et plus complexe que le Danemark, avec des partenaires sociaux plus faibles. Ce que les Danois ont réalisé grâce à un dispositif relativement simple, qui repose très largement sur des prélèvements obligatoires très lourds ${ }^{40}$, la France doit chercher à le faire grâce à une autre combinaison : un mélange de financement et de technique juridique. Elle a commencé, sans en avoir conscience, à le faire.

\section{LA MISE EN OEUVRE DE LA FLEXICURITÉ}

Si l'on est optimiste, on peut dire que la position «d'initiative stratégique» se trouve du côté de la Sécurité sociale professionnelle. En effet, même lorsque celle-ci ne se trouve pas à l'ordre du jour, par exemple dans les lois Aubry de 1998 et 2000, on glane quelque élément qui en conforte le projet ${ }^{41}$. Ce progrès masqué - il vaudrait mieux parler de premiers balbutiements - (A) dessine les linéaments de ce que pourrait être un système d'ensemble. Mais il est au vrai tout aussi possible que l'on s'oriente vers une érosion indéfinie de la stabilité de l'emploi (B).

$405 \%$ du PIB (produit intérieur brut) consacré aux politiques de l'emploi, contre environ 3,5 \% en France.

${ }^{41}$ Développement du compte-épargne-temps, réforme du temps partiel...

\section{L'acquis français}

Les évolutions rattachables à une forme française de flexicurité peuvent être classés en cinq catégories : regain de la liberté professionnelle, financement des transitions, organisation juridique des transitions, validation d'activités hétérogènes en vue de la protection sociale, redéfinition des identités professionnelles.

\section{Le regain de la liberté professionnelle}

La maîtrise par les salariés de leurs carrières implique d'abord que ceux-ci bénéficient de la liberté professionnelle. C'est dans ce domaine que les progrès ont été les plus sensibles. Les courants d'idées opposés - libéraux et «régulateurs»convergent ici en effet.

Il convient ainsi, en premier lieu, de rappeler le recul constant, depuis une quinzaine d'années, des clauses restrictives de la liberté du travail : recul des clauses de non-concurrence ${ }^{42}$, disparition de l'idée selon laquelle le contrat de travail ferait naître une obligation de «fidélité » (Rouast et Durand, 1957). Tout au contraire, la clause d'exclusivité, comme la clause de non-concurrence, doit être justifiée par un intérêt légitime de l'entreprise ${ }^{43}$.

Il ne suffit pas que le salarié puisse librement démissionner ou compléter ses revenus par un autre emploi. L'idée se fait également jour qu'il doit pouvoir sans délai quitter un emploi moins bon - à durée déterminée - pour un meilleur - sous contrat à durée indéterminée ${ }^{44}$.

\footnotetext{
42 Soc. 14 mai 1992, B. n 309 (exigence d'un intérêt légitime de l'entreprise); Soc. 10 juill. 2002, DS 2002.954, note R. Vatinet (exigence d'une contrepartie financière); Soc. 31 mai 2006, Liaisons Sociales, J 963, p. 1 (nullité de la clause qui ne prévoit de contrepartie qu'en cas de rupture à l'initiative de l'employeur); Soc. 15 nov. 2006, LS, J 984, p. 1 (la contrepartie ne doit pas être dérisoire).

${ }^{43}$ Soc. 11 juill. 2000, B. $n^{\circ} 276$ et 277. En outre, la clause d'exclusivité est interdite dans le cadre d'un contrat à temps partiel. Elle est inopposable au salarié-créateur d'entreprise (art. L 121-9 C. trav., L. du $1^{\text {er }}$ août 2003).

${ }^{44}$ Art. L 122-38 C. trav. (L. du 17 janv. 2002). Cette disposition généralise une solution inaugurée par la loi du 16 oct. 1997 à propos des emplois-jeunes (art. L 322-4-20 C. trav.). V. aussi not. la possibilité de rupture anticipée du contrat d'apprentissage en cas d'obtention du diplôme (art. L 115-2 C. trav.) et la possibilité pour l'apprenti de moins de 16 ans de démissionner pour reprendre sa scolarité (art. L 117-17 C. trav.).
} 
Sur un mode mineur, le droit du travail se soucie de plus en plus fréquemment de permettre à un salarié à temps partiel de s'engager dans une seconde activité : interdiction des clauses d'exclusivité, possibilité de refuser une modification d'horaires dont l'éventualité est prévue au contrat ${ }^{45} \ldots$

\section{Le financement des transitions}

Une idée maîtresse du rapport Supiot est de mettre en place en faveur des travailleurs des «droits de tirage sociaux $»^{46}$, que ceux-ci peuvent mobiliser lorsqu'ils en ont besoin pour financer une période d'activité non autrement rémunérée.

Cette idée prend appui :

1. Sur le développement dans plusieurs pays européens de «comptes individuels de temps ». Au lieu d'un décompte hebdomadaire ou mensuel, le compte individuel de temps peut ouvrir à l'individu un « droit de tirage », c'est-à-dire un droit à rémunération, différé dans le temps. Une fois que ce compte existe, pourquoi ne pas admettre que d'autres acteurs que le salarié et l'employeur - par exemple des acteurs publics, dans le cadre d'une politique de soutien aux familles, ou de formation professionnelle - «abondent» le compte ? Le compte-épargnetemps français ${ }^{47}$ constitue une amorce de « droit de tirage social ».

2. Sur le développement de «droits à » versement par la collectivité de revenus pendant une période d'activité non lucrative. Le mécanisme de financement du congé individuel de formation ${ }^{48}$ en fournit une illustration remarquable.

À la différence des dispositifs traditionnels d'assurance et d'assistance, les droits de tirage sociaux sont

45 Art. L 212-4-3, al. 6 C. trav. (possibilité de refuser une modification d'horaire prévue au contrat en raison de son incompatibilité avec « une période d'activité fixée chez un autre employeur ou avec une activité professionnelle non salariée»). Art. L 223-7 C. trav. (établissement de l'ordre des départs en congés en tenant compte d'une autre activité salariée).

46 P. 90.

47 Art. L 227-1 C. trav., assoupli en dernier lieu par la loi du 31 mars 2005.

${ }^{48}$ Art. L 931-8-2 C. trav. Le revenu est versé par un organisme qui perçoit des cotisations patronales et «mutualise » leur utilisation (c'est-à-dire peut accorder à l'un ce qui a été versé au titre de l'emploi de l'autre).
« proactifs »: ils permettent au travailleur d'organiser son avenir.

\section{Les techniques juridiques de coordination}

Pour organiser les transitions, il est souvent nécessaire d'articuler deux situations juridiques, de façon à éliminer les solutions de continuité ou à réduire leurs effets. On doit ici se borner à donner des exemples :

a) Le contrat de travail joue le rôle d'un filet de sécurité qui permet au salarié de s'engager dans une activité nouvelle sans perdre sa position de départ. C'est la multiplication des possibilités de suspension de droit du contrat, avec réintégration au terme de la période de suspension : congé de formation, congécréation d'entreprise ${ }^{49}$, congé de solidarité internationale, congé parental d'éducation... Ou la suspension d'un commun accord du contrat à durée indéterminée pour la durée d'un apprentissage ${ }^{50}$.

b) La juxtaposition de situations, organisée dans un " contrat-cadre », permet d'articuler des activités :

- concomitantes et à temps partiel: c'est l'«employeur recomposé » des groupements d'employeurs ${ }^{51}$, des coopératives utilisées pour l'emploi en commun de main-d'œuvre ${ }^{52}$ ou du travail à temps partagé ${ }^{3} \ldots$, ou encore l'agent public recruté pour une durée supérieure aux besoins de son employeur et mis à la disposition d'une entreprise pour le surplus ${ }^{54}$.

${ }^{49}$ La suspension à temps partiel du contrat de travail (art. L 12232-12 C. trav., L. du $1^{\text {er }}$ août 2003) instituant le cas échéant une forme de pluriactivité. Sur la question en général, V. L. Casaux, La pluriactivité, Thèse Toulouse 1991 ; LGDJ 1998.

${ }^{50}$ Art. L 115-3 C. trav.

${ }^{51}$ Art. L 127-1 s. C. trav. Depuis la loi du 23 fév. 2005 (art. L 12710 à $13 \mathrm{C}$. trav.), ceux-ci peuvent être constitués entre personnes privées et collectivités territoriales. Sur la convention collective alors applicable, RM, JOAN Q. 4 janv. 2007, $\mathrm{n}^{\circ} 17565$, p. 32 ; Bref social, 26 janv. 2007, p. 3.

${ }^{52}$ La loi du 2 août 2005 permet aux groupements d'employeurs de prendre la forme de coopératives, et à certaines coopératives existantes de jouer le rôle de groupements d'employeurs. La loi du 5 janvier 2006 (art. 58, IV) étend cette possibilité aux coopératives d'utilisation de matériel agricole (CUMA) (art. L 127-1 C. trav.).

${ }^{53}$ Art. L 124-24 s. C. trav. $C f$. P.-Y. Verkindt, « Le travail à temps partagé » (L. n 2005-882, 2 août 2005) ou « Pourquoi faire simple quand », JCP S, 1118.2005.

${ }^{54}$ Art. 61, L. n 2005-157 du 23 fév. 2005 (Liaisons sociales, D2 205). 
- successives et de durée limitée, comme le contrat de transition professionnelle, que doivent proposer aux salariés visés par un licenciement économique certaines entreprises ${ }^{55}$.

Ces exemples constituent en somme des versions minimalistes du contrat d'activité du rapport Boissonnat.

c) Le "tuilage» d'une activité dans sa phase initiale par une activité antérieure permet au travailleur de bénéficier, dans la phase critique, d'une protection que les ruptures franches interdiraient. Trois exemples :

- Le contrat d'appui au projet d'entreprise ${ }^{56}$ permet au créateur d'entreprise, personne physique - souvent un ancien salarié - de bénéficier pendant la phase de démarrage, à la fois de l'aide technique et du crédit de l'accompagnateur, mais aussi d'une protection sociale prise en charge par celui-ci ${ }^{57}$. Le créateur d'entreprise agit sous l'ombre tutélaire d'une entreprise plus grande ;

- Chaque fois qu'il est question de reclassement externe, les obligations que supporte l'employeur en cas de licenciement économique font jouer à l'emploi de départ le rôle de point d'appui pour en obtenir un autre. Le «tuilage» est très apparent lorsqu'un employeur, dans le cadre d'un «plan de sauvegarde de l'emploi », recourt à l'outplacement (reclassement externe) ou prête certains salariés à des entreprises avoisinantes en comptant sur le fait qu'une partie d'entre eux sera recrutée définitivement. On pourrait aussi bien concevoir qu'une grande entreprise s'entende avec d'autres pour organiser la «deuxième carrière » de ses salariés âgés ${ }^{58}$;

\footnotetext{
${ }^{55}$ Ordonnance $\mathrm{n}^{\circ}$ 2006-433 du 13 avr. 2006, JO 14 avril 2006 ; ratification par l'article 49 de la loi du 30 déc. 2006. Liaisons Sociales, D4 425 (art. 4 : «Le contrat de transition professionnelle est conclu pour une durée de douze mois. Ce contrat a pour objet l'organisation et le déroulement d'un parcours, qui peut comprendre des mesures d'accompagnement, des périodes de formation et des périodes de travail pour le compte de tout employeur à l'exception des particuliers. »)

${ }^{56}$ Art. L 127-1 s. C. com. (L du 1 $1^{\text {er }}$ août 2003).

${ }^{57}$ Art. L 783-1 s. C. trav.

${ }^{58}$ N'est-ce pas ce que fait jusqu'à un certain point l'Armée, en orientant vers une deuxième carrière civile les ex-militaires de carrière ? Cf. La reconversion des militaires, Rapport AN Dasseux et Martin, $n^{\circ} 3192,2006$.
}

- Le «congé de mobilité», institué par la loi du 30 décembre $2006^{59}$, modalité de «reclassement prévisionnel », permet que soient intercalées des «périodes de travail» en dehors de l'entreprise durant ce congé, alors parfois « suspendu ». Conservant pour un temps son contrat de travail initial, le salarié en conclurait pourtant un second avec l'entreprise extérieure...

\section{La validation d'activités hétérogènes en vue de la protection sociale}

Pour que les changements d'activité soient acceptables, il ne faut pas qu'ils entraînent de conséquence négative dans le domaine de la protection sociale ou de la retraite. C'est l'un des sens qui s'attache, dans le rapport Supiot, à la notion d' '« état professionnel des personnes » : que soient prises en compte et validées comme relevant de l'« état professionnel» des activités diverses de travail - peu importe qu'elles aient eu lieu à titre salarié ou indépendant, public ou privé, lucratif ou non lucratif.

Le droit de la Sécurité sociale a fait - sur le papier des pas en ce sens, par exemple en prévoyant une « demande unique de retraite» pour la liquidation des droits à pension acquis auprès de différents régimes ${ }^{60}$, ou encore en « proratisant » certains planchers de cotisation ${ }^{61}$. Mais on est encore loin du compte. Ainsi, dans le système de retraite complémentaire des salariés AGIRC-ARRCO (Association générale des institutions de retraite des cadres/Association pour le régime de retraite complémentaire des salariés), les droits à retraite ne peuvent pas être liquidés avant 65 ans si l'intéressé est devenu indépendant au moment de sa cessation d'activité6 ${ }^{2}$.

\footnotetext{
${ }^{59}$ Loi n $^{\circ}$ 2006-1770, JO p. 20210 ; art. L 320-2-1 nouveau C. trav. À rapprocher, bien évidemment, du congé de reclassement (art. L 321-4-3 C. trav.).

60 D. n 97-1001 du 27 oct. 1997 (art. R 173-4-1 CSS) ; Circ. 98/ 316 du 4 juin 1998 ; Circ. CNAV 36/98 du 4 juin 1998. Cette mesure s'applique aux pensions de réversion. V. aussi le rattachement à un seul régime de pluriactifs non salariés par la L. du 23 fév. 2005 (art. 64).

61 V. not. art. L 612-4 CSS (L. du 1 ${ }^{\text {er }}$ août 2003) (proratisation des cotisations d'activité non salariée, non agricole en deçà d'un nombre de jours par année civile fixé par décret). Le « coût d'entrée » pour les pluriactifs est ainsi limité.

${ }^{62}$ En dernier lieu, art. 1, accord du 13 nov. 2003. La condition d'avoir fait liquider une pension de retraite à taux plein auprès du régime général écarte ceux qui ont cessé d'être salariés en cours de carrière.
} 


\section{L'identité professionnelle}

Lorsque Alain Supiot (1999) a choisi le terme d'« état professionnel », il a créé une très évocatrice analogie avec la notion d'état-civil. L'état-civil, comme chacun sait, ne se borne pas à conserver la mémoire technique des « données ». L'état-civil est un élément de définition de l'identité des personnes, qu'il inscrit dans un lignage, auxquelles il attribue un patronyme, qu'il rattache à un sexe et à une génération...

L'《 état professionnel » pourrait porter témoignage de l'identité professionnelle. Les «états $»^{63}$ de la société traditionnelle ont été absorbés par le marché et les relations contractuelles. L'identité de substitution qui résultait de la stabilité de l'emploi (« Je suis ajusteur P2 chez Hispano-Suiza... ») a été ébranlée par les remises en question des dernières décennies. On aimerait que l'identité professionnelle puisse être définie indépendamment du contrat de travail ; cela aussi faciliterait les transitions. Force est de constater que le dispositif juridique qui se rapproche le plus de cela, la «validation des acquis de l'expérience», est autrement orienté, puisqu'il conduit à l'attribution de diplômes ou d'éléments de diplômes ${ }^{64}$. Si l'on savait certifier le contenu d'une carrière (en y incluant, bien entendu, les activités non salariées ou à but non lucratif) et les aptitudes acquises, on n'aurait pas besoin de recourir à cet artifice ${ }^{65}$.

\section{Le futur prévisible : une érosion sans rupture}

Vu sous l'angle de la Sécurité sociale professionnelle, l'ANI (accord national interprofessionnel) du

\footnotetext{
${ }^{63}$ Qu'est-ce qu'un état? Non point seulement un statut, mais plutôt la fusion d'une identité, d'un statut et d'une activité (le Rouge et le Noir: l'état d'officier, "Le bel état de prêtre qui mène à tout... »). D'où le succès de la formule d'Alain Supiot. $C f$. Gaudu, « Travail et activité », DS 97.119.

${ }^{64}$ Art. L 900-1 C. trav. (L. du 17 janv. 2002). Cf. Liaisons sociales, D1 265.

${ }^{65} \mathrm{Il}$ faut valider les acquis de l'expérience. Mais s'il est une prérogative qui doit revenir aux métiers (et aux territoires), non aux institutions académiques, c'est bien celle-là. L'objet du diplôme n'est pas de reconnaître une " identité professionnelle »; les exigences scolaires, par ailleurs légitimes, conduisent à refouler une partie importante de ceux qui devraient être « reconnus ». En présence d'une troupe nombreuse en mal de définition de soi, on donne un galon supplémentaire à $5 \%$ du corps des sous-officiers : c'est à côté de la question.
}

11 janvier 2008 sur la modernisation du marché du travail fait penser au classique "pâté d'alouettes » : un peu de sécurisation des trajectoires (avec notamment la généralisation d'une indemnité légale de licenciement portée à un cinquième de mois de salaire par année de présence, dès que le salarié à un an d'ancienneté, l'abrogation prévue du CNE, et surtout avec la «portabilité » de certains droits $\left.{ }^{66}\right)$; davantage d'assouplissement du droit de la rupture du contrat de travail, avec l'extension des possibilités de rupture conventionnelle du contrat de travail et un nouveau contrat à durée déterminée, le « contrat à objet défini ». Si l'on tient compte d'autres projets en cours de réalisation, il est possible de dégager trois axes d'évolution: le contrôle du motif du licenciement, bien qu'écorné, demeure; il en va de même des obligations de reclassement, même si certaines d'entre elles pourraient encore faire l'objet de réformes ; il sera plus facile qu'auparavant de négocier la renonciation du salarié à contester la rupture du contrat de travail.

\section{Les variations sur la motivation du licenciement}

«La rupture $d u$ contrat de travail doit être motivée », formule parfois utilisée par l'entourage du Président de la République ${ }^{67}$ et reprise par l'ANI de 2008, peut sembler ambiguë. Car, entre les deux notions, que le motif du licenciement doive être énoncé par l'employeur, et que ce motif fasse l'objet d'un contrôle judiciaire, il peut y avoir plus que des nuances. Par exemple, l'énonciation du motif pourrait n'avoir pour incidence, limitée, que de permettre de vérifier que le licenciement n'est pas discriminatoire...

Dans ses propositions, le Medef (Mouvement des entreprises de France) a tenté d'avancer dans plusieurs directions, qui tendaient toutes à évincer le contrôle judiciaire : proposition d'une «période de validation économique » du contrat à durée indéterminée, faisant suite à la période d'essai, pendant laquelle la rupture (ou du moins la rupture pour motif

\footnotetext{
${ }^{66}$ Maintien de la couverture complémentaire santé pendant une certaine durée après le licenciement; utilisation du «droit individuel à la formation » par les salariés licenciés, qu'ils soient chômeurs ou aient trouvé un nouvel emploi (art. 14).

${ }_{67}$ P. ex. X. Bertrand, Le Nouvel Observateur, 9 juill. 2007.
} 
économique) n'eût point permis au salarié d'agir en justice ; « contrat de projet », à durée indéterminée, mais dont la réalisation de l'objet eût entraîné la rupture.

Ces propositions, en raison de l'opposition des syndicats et des mêmes difficultés juridiques qui ont entraîné l'échec du $\mathrm{CNE}^{68}$, n'ont pas été suivies d'effet. Il en reste, cependant, une apparente généralisation de la période d'essai à tous les contrats à durée indéterminée ${ }^{69}$; un " contrat à objet défini », à durée déterminée certes (18 mois au moins et 36 mois au plus), mais qui peut être rompu à chaque date anniversaire de sa conclusion "pour un motif réel et sérieux»; un assouplissement limité du régime du licenciement ${ }^{70}$.

\section{L'extériorisation du reclassement externe}

Dans son programme présidentiel, le Président de la République promettait : «[...]... je créerai la sécurité sociale professionnelle. [...] Une personne licenciée pour des raisons économiques ne perdra pas son contrat de travail : celui-ci sera transféré au service public de l'emploi qui lui garantira $90 \%$ de sa rémunération antérieure aussi longtemps que nécessaire, pour suivre une formation qualifiante, trouver un nouvel emploi, essayer un nouveau métier. »

Cette question a été laissée de côté dans les négociations qui viennent de s'achever. La raison peut être, non point l'abandon du projet, mais plutôt le fait que celui-ci est subordonné à la réalisation complète de la fusion entre l'ANPE (Agence nationale pour l'emploi) et l'UNEDIC.

Si ce projet doit avoir une suite, que signifie alors la formule énigmatique du «transfert du contrat de travail au service de l'emploi»? Littéralement, elle n'a guère de sens. Les commentateurs y ont parfois vu une promesse de généralisation du « contrat de transition professionnelle », évoqué plus haut. Elle fait aussi penser à une pratique courante en Allemagne.

\footnotetext{
${ }^{68}$ Cf. supra, $\mathrm{n}^{\circ} 18$.

${ }^{69}$ Actuellement, la loi permet, mais n'impose pas la stipulation d'une période d'essai (art. L 122-4 C. trav.).

${ }^{70}$ L'employeur, aujourd'hui lié par le motif qu'il a énoncé dans la lettre de licenciement et condamné s'il n'en énonce aucun, pourrait a posteriori invoquer un motif ; les dommages-intérêts en cas de licenciement sans cause réelle et sérieuse seraient plafonnés.
}

Les entreprises allemandes supportent une assez stricte obligation de reclassement interne des salariés menacés de licenciement. En revanche, la loi allemande n'impose pas d'obligation de reclassement externe. Toutefois, l'équivalent allemand du plan de sauvegarde de l'emploi (Sozialplan, plan social) doit faire l'objet d'une codécision entre l'employeur et le conseil d'établissement (Betriebsrat). Si elle n'est pas obligatoire, la contribution de l'entreprise au reclassement externe peut alors être prévue par un accord d'établissement conclu entre l'employeur et le Betriebsrat. Dans le cadre des plans sociaux codécidés, il n'est ainsi pas rare que les salariés dont l'emploi est supprimé voient leurs contrats transférés à un «employeur de transition $»^{11}$. Il y a donc bien, comme dans la formule utilisée par le Président de la République, maintien du contrat de travail pendant un temps, mais pas avec l'employeur initial, qui est par ailleurs soumis à un prélèvement obligatoire afin de financer le dispositif.

Si l'on transpose ce système en France, on pourrait imaginer que l'entreprise qui licencie pour motif économique soit déchargée de l'obligation de prendre des mesures de reclassement externe, moyennant un coût forfaitaire. Il n'est pas certain que les travailleurs intéressés perdent au change, à condition - hypothèse optimiste - que l'obligation de reclassement interne, actuellement imposée par la loi, soit en revanche strictement conservée.

\section{L'essor de la "séparabilité »}

Le terme de séparabilité relevait jusqu'à présent de la physique quantique, et non des politiques sociales. Il fait son apparition dans un «Livre blanc» rendu public par le Medef en janvier 2007. L'idée générale est d'assouplir les conditions de la rupture d'un commun accord du contrat de travail (car celle-ci, en réalité, existe déjà).

Il existait jusqu'à présent deux formes de rupture d'un commun accord du contrat de travail : d'une part, la loi prévoit, dans plusieurs hypothèses, que lorsque le salarié accepte une modalité de reclassement proposée par l'employeur dans le cadre d'une

\footnotetext{
71 Transfergesellschaft (société de transition). V. Liaisons sociales Europe, $\mathrm{n}^{\circ} 120,20$ janv. 2005.
} 
procédure de licenciement pour motif économique, le contrat est « rompu d'un commun accord» : anciennes «convention de conversion», actuels «congés de mobilité » (article L 320-2-1 du Code du travail), « conventions de reclassement personnalisé » (article L 321-4-2 du Code du travail) et contrats de transition professionnelle, déjà évoqués ${ }^{72}$. En dehors de ces hypothèses légales il $\mathrm{y} \mathrm{a}$, conformément au droit commun des contrats, rupture d'un commun accord chaque fois que le salarié et l'employeur tombent d'accord sur le principe de la rupture. Toutefois, il faut que le salarié ait, autant que l'employeur, voulu la rupture ${ }^{73}$. Ainsi, lorsque le salarié, mis devant le fait accompli d'une décision de l'employeur de rompre son contrat, se voit proposer une « rupture d'un commun accord », il y a de grandes chances que les juges qualifient la rupture de licenciement.

C'est précisément en cela, peut-on penser, qu'innove l'accord du 11 janvier 2008. En effet, les modalités prévues (possibilité pour le salarié de se faire assister lors des discussions préalables, de prendre contact avec le service public de l'emploi pour envisager la suite de son parcours professionnel, droit de rétractation, homologation de la convention par le directeur départemental du Travail) évoquent la situation d'un salarié qui négocie après que l'on lui a au préalable annoncé la suppression de son emploi. La rupture conventionnelle est construite comme un acte de renonciation $d u$ salarié, et les garanties mêmes dont on l'entoure montre qu'il s'agit de lui demander de

\footnotetext{
${ }^{72}$ On ne sait pas d'ailleurs très bien, dans ces hypothèses, si le juge doit néanmoins, à la demande du salarié, contrôler le caractère réel et sérieux du motif (c'est ce qu'il faisait naguère pour les conventions de conversion par application de l'article L 511-1, alinéa 3 du Code du travail), ou s'il doit refuser de le faire.

${ }^{73}$ Hypothèse courante si le salarié, qui a trouvé un emploi plus prometteur, a besoin de se dégager rapidement de ses obligations pour saisir l'occasion. Mais le départ doit être "volontaire» (Cass. Soc. 13 sept. 2005, Bull. civ., V, $\mathrm{n}^{\circ} 252$ ).
}

« consentir» à une rupture qu'il ne souhaite pas. Il est à craindre qu'après la transposition législative, il s'agisse d'une pseudo-rupture d'un commun accord. Les procédures de licenciement économique sont toutefois conservées, tout comme l'exigence d'un motif réel et sérieux de licenciement (mais celui-ci ne se contrôle plus en cas de « rupture conventionnelle »). Après les assouplissements apportés par la loi du 18 janvier 2005, le droit du licenciement subit ainsi une nouvelle érosion, mais limitée.

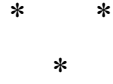

Si l'on veut comparer, l'accord de 2008 fait penser à la loi allemande du 24 décembre 2003, adoptée sous le Gouvernement Schröder juste avant les lois Hartz $^{74}$. En poursuivant la comparaison, l'on s'aperçoit que l'érosion du droit du travail atteint les uns après les autres tous les pays d'Europe : ce qui reste de la semaine de 36 heures est en discussion aux Pays-Bas ; le niveau de l'indemnisation du chômage est remis en question au Danemark. Pas de rupture, mais, peut-être, une spirale descendante commune aux pays d'Europe continentale de l'Ouest. Et c'est dans la voie d'une spirale descendante qu'oriente le «Livre Vert» de la Commission européenne. En effet, au lieu de dégager, comme des alternatives, les différentes politiques concevables, il prend appui sur l'écho favorable qu'ont suscité les expériences hollandaise et danoise pour proposer ce qui ressemble davantage à une déréglementation du droit du licenciement.

\footnotetext{
${ }^{74}$ Raccourcissement des délais de licenciement, multiplication des hypothèses dans lesquelles, à la suite d'un accord de l'employeur avec le conseil d'établissement ou d'une transaction, le salarié ne peut pas agir en justice...
} 
Bibliographie

Algan Y. et Cahuc P. (2005), Civic Attitudes and the design of Labor Market Institutions : Which Countries can Implement the Danish Flexicurity Model?, www.bm.dk/ministeren/taler/050616_uk.asp.

Auroux J. (1981), Les droits des travailleurs, rapport au Président de la République et au Premier ministre, La Documentation française.

Barbier J.-C. (2005), «Apprendre vraiment du Danemark : réflexion sur le "miracle danois" », CEE $05 / 02$.

Barbier J.-C. (2007), « Au-delà de la flex-sécurité, une cohérence sociétale solidaire au Danemark », in Repenser la solidarité au XXIe siècle, (dir. S. Paugam), PUF.

Barbier J.-C., Brygoo A., Viguier F. et Tarquis F. (2002), « Managing labour market-related risks : a comparative analysis of regulation frameworks and policies ", ESOPE Project, Precarious employment in Europe..., rapport français, avril, not. p. 34 s., 40.

Boyer R. (2006), La flexicurité danoise: une référence pour la France? éd. ENS-rue d'Ulm, coll. du CEPREMAP.

Cahuc P., Kramarz F. (2005), Le contrat de travail unique, clef de voûte d'une Sécurité sociale professionnelle, rapport au ministre de l'Économie et au ministre de l'Emploi.

Cahuc P., Kramarz F. (2005), De la précarité à la mobilité : vers une sécurité sociale professionnelle, rapport au ministre de l'Économie et au ministre de l'Emploi, La Documentation française.

Cahuc P. (2003), Pour une meilleure protection de l'emploi, rapport pour la CCIP, mai, p. 14, p. 25 s. et p. 158.

Centre d'Analyse Stratégique (2006), Besoins de main-d'œuvre et politique migratoire, La Documentation française, annexe 2, p. 11.
Commissariat Général du Plan (1995), Le travail dans vingt ans, rapport de la commission présidée par Jean Boissonnat, Odile Jacob-La Documentation française.

Commission des communautés européennes (2006), Moderniser le droit du travail pour relever les défis du XXI siècle, 22 nov., 708 final.

Coutrot T. (1999), « 35 heures, marchés transitionnels, droits de tirage sociaux : du mauvais usage des bonnes idées », Droit social, 99.659.

Gazier B. (2003), Tous sublimes, Flammarion, coll. « essais ».

Gazier B. (1998), «Ce que sont les marchés transitionnels », Cahiers du CEE, 37, p. 339.

Gautié J. (2005), « Les économistes contre la protection de l'emploi : de la dérégulation à la flexicurité », Droit social, 2005.40.

Gaudu F. (2007), La Sécurité sociale professionnelle, un seul lit pour deux rêves », Droit Social, 2007.393.

Gaudu F. (2005), « Des illusions des juristes aux illusions scientistes » in Le droit du travail confronté à l'économie, (dir. A. Jeammaud), Dalloz, Actes.

Gaudu F. (2007), «Un document qui ne tient pas le langage de la vérité », Droit du travail, 2007.75.

Institut de l'entreprise (1998), « Croissance et emploi : pourquoi les Pays-Bas font-ils mieux que la France ? ».

Jackson V.G. (2005), « Employee Representation in the Board Compared : A Fuzzy Sets Analysis of Corporate Governance, Unionism and Political Institutions », Industrielle Beziehungen, 12. Jg., Heft 3.

Le Duigou J.-C. (2005), « La sécurité sociale professionnelle : une utopie réaliste », in CGT, Analyses et documents économiques, $\mathrm{n}^{\circ} 98$, février, p. 48

Lojkine J. (1999), « À propos du rapport Supiot », Droit social 99.669. 
Rouast A. et Durand P. (éd. 1957), Précis Dalloz de droit du travail, $\mathrm{n}^{\circ} 331$.

Schmidt G. (2002), Wege in eine neue Vollbeschäftigung (Chemins d'un nouveau plein emploi), Campus Verlag.

Supiot A. (1999) (dir.), Au-delà de l'emploi, rapport pour la DGV de la Commission européenne, Flammarion.
Thibault B. (2002), « Sécurité, travail et solidarité », NVO 5 avril, p. 1.

Visser J. (1997), A Dutch Miracle. Job Growth, Welfare Reform and Corporatism in the Netherlands, Amsterdam University Press.

Visser J. (1999), The First Part-time Economy in the World. Does it Work?, Japan Productivity Center for Socio-Economic Development, Tokyo, 24-25 mars.

\section{Résumé}

\section{De la flexicurité à la sécurité sociale professionnelle. L'emploi entre mobilité et stabilité François Gaudu}

L'article tente de présenter le débat français sur la réforme du droit du travail dans le contexte européen. Sous les appellations respectives de "Sécurité sociale professionnelle » et de flexicurity, deux débats au contenu très proche se déroulent dans le cadre national français et dans le cadre européen. Nous étudions ici d'abord les stratégies de flexicurité. Nous envisageons ensuite les réalisations qui, quoique de façon balbutiante, correspondent à une première mise en œuvre pratique en France. Des deux possibilités - spirale descendante ou émergence d'un nouveau système conforme à la tradition de l'Europe continentale de l'Ouest -, il n'est pas possible aujourd'hui de dire laquelle l'emportera.

\section{Mots clés}

Sécurisation des parcours professionnels, législation du travail, contrat de travail, comparaison internationale, Europe

Journal of Economic Literature : K 31 - Labor Law ; M 55 - Labor Contracting Devices ; J 53 - Labor Management Relations; Industrial Jurisprudence 